821.131.1.09-31

https://doi.org/10.18485/italbg.2018.1.6

Karol Karp*

Università Niccolò Copernico di Toruń

\title{
IL VIAGGIO IN ALBANIA. IL TUO NOME È UNA PROMESSA DI ANILDA IBRAHIMI
}

\begin{abstract}
L'articolo analizza il tema del viaggio nell'ultimo romanzo di Anilda Ibrahimi, una scrittrice migrante d'espressione italiana, ed è diviso in tre parti. Ogni parte è dedicata a un viaggio diverso. I protagonisti si spostano dall'Occidente in Albania per raggiungere mete come Tirana, Kavajë e Valona. La disamina indaga diversi aspetti del viaggio, si concentra sulle motivazioni dei viaggiatori, sulla loro condizione, sull'immagine della nuova realtà, nonché sullo stesso atto del viaggiare. Il viaggio svolge un ruolo molto importante, consente di acquistare informazioni sulla storia e sulla cultura, di formulare giudizi sul passato e addirittura di evitare la morte. L'Albania viene presentata in modo positivo, si dimostra un paese dove il soggetto può sperare bene per il futuro, nonostante alcuni momenti siano difficili.
\end{abstract}

Parole chiave: Italia, Albania, viaggio, ospitalità, cultura, autocoscienza.

\section{INTRODUZIONE}

Il viaggio è uno dei temi importanti che trapelano dalla produzione degli scrittori d'espressione italiana e origine albanese. Vale la pena rilevare che nel volume collettaneo Il confine liquido. Rapporti letterari e interculturali fra Italia e Albania (2013), curato da Daniele Comberiati ed Emma Bond, sono analizzate alcune delle loro opere, tra le quali si può citare Rosso come una sposa (2008) di Anilda Ibrahimi.

La scrittrice, nata a Valona nel 1972, da anni vive in Italia. Con il suo ultimo romanzo ${ }^{1}$ Il tuo nome è una promessa (2017), fornisce una nuova prospettiva della problematica odeporica nell'ampio panorama della lettera-

*karol_karp@vp.pl

${ }^{1}$ Il titolo dell'opera allude a un elemento della cultura albanese, alla cosiddetta besa, cioè parola data, concetto istituito dal Kanun, un codice culturale antico, che vige in alcune parti del paese fino ai nostri giorni. Besa è il nome che i protagonisti danno alla loro figlia per nascondere la sua origine ebrea e salvarla così dall'oppressione tedesca. 
tura prodotta dai suoi connazionali, dove di solito ${ }^{2}$, e come esempi possiamo addurre Va e non torna (2000) di Ron Kubati oppure Senza bagagli (1997) di Elvira Dones, lo spostamento va dall'Albania in Occidente. In Ibrahimi invece la meta del viaggio costituiscono diverse località albanesi.

L'opera $^{3}$ è narrata in terza persona e contiene due piani temporali abbastanza lontani l'uno dall'altro. Il primo ingloba i tempi odierni, il secondo invece va dagli anni Trenta fino ai primi anni Novanta del Novecento. La tecnica di imperniare la narrazione su due piani temporali diversi risulta abbastanza frequente nei testi prodotti da autori migranti di origine albanese. La si percepisce ad esempio in romanzi come: Senza bagagli di Dones, Va e non torna oppure $M$ (2002) di Kubati.

L'obiettivo del presente articolo è quello di analizzare il tema del viaggio inteso nella sua accezione ampia. Le nostre riflessioni ingloberanno non solo la specificità dello stesso atto del viaggiare, ma anche le motivazioni dei protagonisti, la loro condizione nella nuova realtà, i cambiamenti che avvengono nella loro vita.

\section{DA NEW YORK A TIRANA}

Il romanzo si apre con la descrizione della figura di Rebecca, una donna moderna in carriera, rappresentante di un'organizzazione internazionale, che giunge nella capitale albanese per lavoro, lasciando la figlia, il marito e la madre in America. Appare come una persona molto coraggiosa ed emozionante, propensa ad accettare diverse sfide. Il viaggio che compie è lungo e solitario, la destinazione è un paese povero, dove le donne straniere, fisicamente diverse da quelle locali, rischiano di diventare oggetto di abusi.

Nei testi degli autori migranti viene rilevato non di rado come l'aspetto esteriore e la provenienza provochino problemi alle donne albanesi che giungono in Italia. Nell'opera Il paese dove non si muore mai (2005) di Ornela Vorpsi la figura femminile vive una profonda delusione. Dopo 1'arrivo sull'altra sponda dell'Adriatico agli occhi degli autoctoni non è che una putana. La Vorpsi parla degli stereotipi sulle donne albanesi diffusi in

\footnotetext{
${ }^{2}$ Nel romanzo di Leonard Guaci intitolato La panciera rossa (1999), vincitore del premio letterario Antonio Sebastiani, il viaggio è compiuto in Albania da una donna italiana.

${ }^{3}$ Il testo è transgenerico, vi si mescolano elementi tipici del romanzo di viaggio, di quello storico, psicologico, di ambiente e costume. La scrittrice si muove con abilità tra $\mathrm{i}$ vari generi e i loro singoli elementi formano un tutto omogeneo, sono ben saldati. Lo stile è scorrevole e risente di una grande precisione. I valori del romanzo sono stati di recente riconosciuti dalla critica italiana. Con Il tuo nome è una promessa Ibrahimi ha vinto la trentatreesima edizione del prestigioso premio letterario Rapallo Carige.
} 
Italia, cercando di mettere in questione la loro correttezza. Ibrahimi invece intende confutare lo stereotipo dell'Albania, considerata nella cultura occidentale come una terra pericolosa. La sua protagonista non vi cade vittima di una qualsiasi violenza. Inoltre, fin dall'inizio prova una certa confidenza nei suoi confronti. Intraprendendo il viaggio mira non solo a realizzare gli scopi professionali, ma intende anche conoscere bene la dimensione che costituisce una parte importante della storia familiare. Sua madre ha vissuto in Albania in passato. Sulla decisione che prende influisce anche la relazione problematica con il marito. Il viaggio le consentirà di capire se lo ami ancora, forse marcherà una nuova tappa nella sua vita. Lasciando gli Stati Uniti, la donna di sicuro si nutre di speranza.

Nel romanzo Vergine giurata (2007) della Dones la protagonista parte dall'Albania negli Stati Uniti. La direzione è dunque opposta a quella del viaggio intrappreso da Rebecca, ma lo scopo risulta senz'altro simile. In America Hana riesce a distanziarsi dalla realtà in cui non si sentiva a suo agio in quanto costretta dal codice culturale, dal cosiddetto Kanun, sempre in vigore in alcune parti dell'Albania, a camuffare la sua vera identità sessuale. Come in Ibrahimi, nel nuovo paese la donna ottiene la possibilità di sperare bene per il futuro, di iniziare una nuova tappa nel suo cammino esistenziale. Hana mira a riacquistare la femminilità negatale in terra natia. La sua riscoperta è un lungo processo che senz'altro le consente anche di conoscere meglio se stessa. Dones dedica attenzione al nuovo rapporto che per via del viaggio si instaura tra la protagonista e la sua autocoscienza. In Ibrahimi invece la prospettiva è più esteriore. Accolta dalla realtà albanese, Rebecca, prova una specie di ammirazione. Con avidità si immerge nella capitale albanese, nella sua architettura, nei suoi colori e odori, riceve gli impulsi visivi e olfattivi che ne vanno verso la sua direzione.

Il suo rapporto con i locali è caratterizzato dalla cordialità e dall'armonia. Fra essi il ruolo più importante svolge l'assistente Andi, con cui vive una storia d'amore. Quest'esperienza permette di capire meglio la natura della protagonista, dà prova della sua propensione al tradimento. Col tempo il lettore si rende conto che Rebecca considera Andi soltanto un amico con cui piacevolmente passa il tempo ${ }^{4}$. In tal modo il viaggio si rivela portatore di libertà, fa sparire certi limiti caratteristici nella realtà del proprio paese,

\footnotetext{
${ }^{4} \grave{E}$ opportuno rilevare che dai discorsi dei protagonisti trapela il tema del viaggio. Si recano insieme da un luogo all'altro all'interno del paese, usando la macchina, e commentano le varie caratteristiche dello spostamento, tra cui la sua lunghezza. Hanno opinioni diverse su cosa significhi un lungo viaggio, che risultano dalla loro provenienza e dalle esperienze vissute. Rebecca associa il lungo viaggio agli spazi americani, in Albania - sembra dire - vista la sua superficie, il viaggio non può essere lungo. Ibrahimi attesta come sia fluida la nozione di viaggio e come sia individuale la sua percezione.
} 
in un certo senso, garantisce l'anonimato e facilita relazioni interpersonali. Nel romanzo risulta molto forte il legame tra viaggio e tradimento. $\mathrm{Nel}$ caso di Rebecca viaggiare molto spesso significa tradire. Esso si vede bene anche nei suoi ricordi di un viaggio in Svezia. Attraverso il tema del viaggio Ibrahimi costruisce l'immagine di una donna peccatrice, che però non suscita disgusto e perplessità. Paradossalmente non le si può negare il diritto di compiere azioni trasgressive in quanto sono condizionate dai suoi bisogni interiori individuali.

In Ibrahimi la tecnica di tematizzare l'arrivo e la permanenza in un paese straniero è molto diversa dalla tendenza generale diffusa nei romanzi prodotti da scrittori italofoni e incentrati sulle migrazioni nell'epoca contemporanea. Ad esempio nell'opera Madre piccola (2007) di Cristina Ali Farah ci imbattiamo nelle figure di immigrati somali in Italia, che diventano destinatari di ostilità e pregiudizi per via dell'atteggiamento degli autoctoni. In una posizione esistenziale problematica degli immigrati in Italia ci inoltriamo anche con romanzi di Amara Lakhous come Lo scontro di civiltà per un ascensore a piazza Vittorio (2006) oppure Divorzio all'islamica a viale Marconi (2010).

L'armonia che si crea tra la protagonista e la realtà albanese deriva dal suo status economico e dalla provenienza, nonché dalle caratteristiche dello stesso viaggio che ha compiuto, e attraverso cui non palesa la necessità di scappare dalla povertà oppure dall'oppressione politica, ma il desiderio di realizzare scopi personali.

La condizione di Rebecca in Albania attesta come al suo viaggio sia legata l'illusione. Risulta d'obbligo subito precisare però che essa lo caratterizza in modo parziale. Da una parte il viaggio le impedisce di distanziarsi completamente dal passato, dall'altra è portatore di cambiamenti significativi, di nuove informazioni. A proposito del legame tra viaggio e illusione la stessa Ibrahimi (2017: 140) dice:

Quell'illusione che si ha quando si cambia luogo. Di poter annullare il tempo. Pensare che quel modo che hanno il giorno e la notte di susseguirsi sia casuale. Come lo è tutto ciò che accade all'alba o al crepuscolo, dai grandi amori alle storie insignificanti che si cancellano il giorno dopo con il cambio delle lenzuola. L'illusione di cominciare un'altra vita. Di concedersi un nuovo inizio.

Una parte considerevole dei pensieri che popolano l'autocoscienza di Rebecca in Albania riguarda la sua famiglia. Secondo Marc Augé (2009: 91) la capacità umana di dimenticare consente di approfittare appieno del presente. Alla protagonista riaffiorano alla mente i ricordi di Thomas e della figlia Sarah, facendo sì che si renda conto dell'impossibilità di fuggire dal passato, di cancellare le esperienze vissute. Alla luce della teoria di Augé, è giusto affermare che essi potranno costituire una certa barriera nel suo 
sviluppo individuale. Dall'altro lato però, la loro stessa presenza nell'animo di Rebecca fornisce anche un'informazione sul loro carattere. Come dice John Henderson (2005: 101), commentando Freud, l'individuo, nella sua subcoscienza, ha la tendenza a far eliminare i ricordi spiacevoli.

A un certo punto la mancanza delle persone care diventa così forte che Rebecca fa venire Thomas in Albania ${ }^{5}$. La nuova realtà le fornisce senz'altro molte emozioni, sentimenti gioiosi, ma in fin dei conti la protagonista non riesce a eliminare dalla sua interiorità la nostalgia dello spazio vitale americano, per cui cerca con ostinazione di costruire intorno a sé una dimensione che vi fosse simile, che fosse un suo sostituto. Proprio Thomas ne costituirà uno degli elementi. Un altro elemento sarà la casa che vuole "vicino al lago, come quella di New York" (Ibrahimi 2017: 143).

Il viaggio che ha compiuto le consente di capire inanzittutto quanto il marito sia importante nella sua vita. Proprio in Albania inizia a riflettere sulle cause dei problemi emersi nella loro vita coniugale, prendendo coscienza degli errori commessi. Ibrahimi ci presenta la donna che per via di un viaggio piacevole e misterioso cambia il suo atteggiamento verso diverse cose che prima le provocavano dubbio e sgomento, la donna che fa i conti con il proprio passato.

Nel romanzo è rintracciabile una specie di motivo della redenzione. Rinsaldando la relazione con il marito, Rebecca risulta una persona retta, che di sicuro non intende più tradire. Vale la pena mettere in risalto che il motivo della redenzione trapela anche dall'opera di Ingrid Beatrice Coman, una scrittrice migrante di origine romena, intitolata Dodici più un angelo (2012). Vi campeggiano tre figure maschili che inizialmente si immergono nel peccato, ma in fin dei conti riescono a comprendere cosa significa la vita giusta. La loro trasformazione è dovuta all'intervento di un personaggio straordinario, di un angelo chiamato Emanuel, che parla loro in modo diretto di tutta la falsità che li caratterizza. In Coman i peccatori cambiano proprio attraverso il suo atteggiamento, in Ibrahimi invece è il viaggio a fare riscoprire alla protagonista l'importanza del ruolo che lo sposo svolge nel suo spazio esistenziale, la rende propensa a pentirsi di come l'ha trattato.

\footnotetext{
${ }^{5}$ Il viaggio intrapreso dall'uomo traduce soprattutto il suo desiderio di raggiungere la moglie e riparare la loro relazione, ma costituisce anche l'occasione per conoscere l'Albania, che fin dall'inizio gli sembra una terra esotica. Thomas ha anche un altro scopo: intende fare delle ricerche storiche, miranti a ricostruire l'immagine della vita e la presenza degli ebrei in questo paese. È un compito molto ambizioso che lo eccita, desidera realizzarlo da tempo. "Thomas si trova davanti alla storia che cerca da sempre. Deve andare da tutti, porta a porta, in ogni villaggio, in ogni città. Parlare con loro, raccogliere i rimasugli di quelle vite. Ricucire come si fa con ago e filo" (Ibrahimi 2017: 210).
} 
In Albania Rebecca acquista un sapere rilevante su varie usanze e su vari elementi culturali diffusi nella società del paese, tra i quali il fenomeno di sehir, riscontrabile anche, occorre dirlo, in altri testi in italiano prodotti da scrittori migranti albanesi. La sua presenza si nota nel romanzo di Ornela Vorpsi La mano che non mordi (2007), nonché in quello di Artur Spanjolli intitolato L'accusa silenziosa (2007). Sehir significa guardare gli altri, osservare la loro vita in modo avido, trarne piacere; è completamente sconosciuto nella cultura occidentale e perciò difficile da capire per gli stranieri. In Vorpsi e in Spanjolli viene sottolineato tra l'altro il suo ruolo negativo, quello di costituire un certo limite alla libertà, un attacco alla privacy. Ibrahimi invece si concentra soltanto sui suoi effetti positivi. Facendo sehir, le vecchie in cui si imbatte la protagonista, si concentrano sulle faccende altrui e ciò consente loro di distanziarsi dai crucci che custodiscono nella propria interiorità.

Sehir lega in un patto le due parti, chi è protagonista e chi assiste. [...] È difficile da far capire a uno straniero. [...] L'unica cosa che unisce queste vecchie alla vita è il sehir che fanno dalla loro finestra. Tutto il resto è dimenticato. Il rancore per la sorte avversa, il dolore per l'inganno della vita, i loro momenti felici, lo spavento per la morte. (Ibrahimi 2017: 84-85)

Rebecca si accorge del ruolo che sehir svolge nella vita delle vecchiette e perciò lo apprezza. Ė molto arrabbiata invece scoprendo un altro costume radicato nella società albanese, il cui carattere la spinge a considerarla maschilista. Il suo disgusto suscita la storia di una ragazza violentata, la cui verginità paradossalmente costituisce il fattore che privilegia il suo oppressore. Secondo il codice culturale l'uomo che ha un rapporto sessuale con una vergine, ha la priorità di prenderla in sposa. La sua forza fisica lo rende dunque capace di realizzare gli scopi matrimoniali e di disporre in modo meccanico del corpo femminile. La protagonista rileva come ciò sia assurdo e manifesta l'intenzione di fornire aiuto alle donne, vittime di violenza in Albania, tramite la sua organizzazione.

Oltre alle informazioni sulle usanze radicate nella cultura, il viaggio compiuto la porta a ricostruire alcuni elementi significativi della storia del paese, senz'altro il frutto di ricerche minuziose effettuate dalla stessa Ibrahimi, come ad esempio, l'autoproclamazione dell'indipendenza nel 1912 e la creazione nel 1914 sul territorio albanese di un principato governato da Guglielmo di Wied, oppure il primo esodo degli ebrei e il loro arrivo a Valona dalla Spagna cattolica nel 1490.

La trama conferma la correttezza della famosa teoria dello studioso americano Hayden White (2010) sul legame tra storia e letteratura. Il contributo di White risiede nel sottolineare la forma letteraria della storia. L'opera è in sintonia con una tendenza generale caratteristica nella narrativa italiana 
contemporanea. Guido Crainz (2007: 95) osserva come vi si riscontrino numerosi motivi storici ${ }^{6}$ inerenti alla nazione italiana. La scrittrice albanese si concentra sulla storia della propria terra natale, a volte però, si riferisce anche all'Italia.

\section{DA BERLINO A KAVAJË}

Nel secondo piano temporale su cui è basata la narrazione del romanzo viene delineata l'immagine di una famiglia ebrea, composta da cinque persone: la nonna Rachel, i coniugi Hadas e Ruth e le bambine: la più piccola Abigail e sua sorella Esther, in futuro la madre di Rebecca, che vive nella capitale tedesca, poi è costretta a scappare per l'atmosfera pericolosa creatasi nel paese. Cercando un riparo, si reca in Albania.

Ibrahimi, attingendo a fonti storiche, rievoca con abilità la condizione degli ebrei in Germania prima dello scoppio della seconda guerra mondiale, sottolinea l'ospitalità che hanno offerto loro le autorità albanesi.

Avevano cercato a tutti i costi un modo per allontanarsi dal paese. L'America, a cui ambivano in tanti, era diventata un miraggio. Le altre ambasciate non rilasciavano più visti agli ebrei. Invece quel piccolo stato [...] nessuno accoglieva ebrei. Il loro re, di nome Zog, salito al potere nel 1928, voleva modernizzarlo e per questo sin dal primo momento aveva invitato tutti gli ebrei ad andarci. I visti venivano rilasciati immediatamente al consolato di Berlino. (Ibrahimi, 2017: 20)

I protagonisti, soprattutto quelli adulti, in quanto consapevoli della minaccia, si spostano intenzionati a evitare la morte, il viaggio costituisce l'unica possibilità di salvare la vita. I sentimenti che lo accompagnano integrano la speranza alla paura, ma in fin dei conti la speranza domina. La paura iniziale è provocata dalla stessa insicurezza relativa a ogni cambiamento ed è una reazione naturale. Come afferma Howard Phillips Lovecraft (1969: 35), la paura è la più vecchia e la più forte emozione umana; la paura più forte nasce da quello che l'individuo non conosce e che pensa di dover conoscere. Col tempo essa sparisce dall'autocoscienza dei Rosen e viene sostituita dal desiderio di gustare la normalità.

La scrittrice pone l'accento sulle caratteristiche dello spostamento, sul fatto che dura molto tempo, porta stanchezza, e a un certo punto forse anche un po' di rassegnazione, di indifferenza. I Rosen non possono emettere un sospiro di sollievo che quando raggiungono la meta, che in quel momento particolare sembra loro la nuova terra promessa, dove potranno trovare migliori condizioni di vita.

${ }^{6}$ Sulla storia nella letteratura italiana novecentesca, si consulti il saggio di Hanna Serkowska (2012). 
Ibrahimi rovescia in modo totale il paradigma diffuso nei testi degli altri autori albanesi d'espressione italiana, in cui la terra promessa è sempre l'Italia, considerata sinonimo di benessere e di numerose possibilità di sviluppo. In opere come Il paese dove non si muore mai di Ornela Vorpsi, I nipoti di Scanderbeg (2012) di Artur Spanjolli oppure I grandi occhi del mare (2005) di Leonard Guaci, i personaggi provengono dall'Albania, nella cui cultura è radicata un'immagine precisa della terra sull'altra sponda dell'Adriatico, inerente senz'altro alla storia delle relazioni italo-albanesi. Ibrahimi effettua un compito straordinario, riesce a evocare informazioni su avvenimenti realmente accaduti di cui fa uso per mostrare l'Albania in una luce molto positiva, completamente diversa da quella nelle opere di altri rappresentanti della letteratura migrante. I suoi protagonisti sono ben accolti nella nuova realtà, non soffrono per le proprie origini, per il fatto di essere stranieri. La loro vita si normalizza, trovano una nuova casa e incontrano persone benevole pronte ad aiutarli.

Il processo di integrazione degli ebrei, come quello di Rebecca, riguarda due dimensioni. Per sentirsi a loro agio, i Rosen devono prendere familiarità con il nuovo spazio e i locali che ne fanno parte integrante. Il viaggio compiuto gli porta la calma tanto desiderata, introduce elementi positivi nella loro vita, dà loro una nuova forza e spinge ad agire per raggiungere la felicità. Non si sentono più sospesi tra la vita e la morte, ma si accingono a impegnarsi in varie iniziative e migliorare la propria sorte.

La loro permanenza in Albania consente al narratore di tratteggiare un quadro interessante della realtà socio-politica del paese ai tempi della seconda guerra mondiale, in cui campeggiano le relazioni italo-albanesi. Le autorità italiane mandano sull'altra sponda dell'Adriatico i soldati per costruire centri per i rifugiati, tra cui quello di Kavajë, dove sono trasportati i Rosen. Secondo i protagonisti gli italiani sono personaggi retti, riescono a instaurare con loro rapporti cordiali. Il viaggio verso Kavajë gli provoca preoccupazioni e un senso di inferiorità. Si ritengono oggetti da spostare. Dalla prospettiva del lettore esso ha un carattere conoscitivo, svela informazioni sulla loro condizione. Sebbene in Albania gustino la normalità, non riescono a sentirsi completamente sicuri. Il sentimento di insicurezza diventa più intenso nel 1943, che segna l'inizio dell'occupazione tedesca. Per via della venuta dei tedeschi, i Rosen si trovano vicino agli oppressori da cui sono fuggiti prima dello scoppio della guerra e l'insicurezza si trasforma a poco a poco in una paura enorme per la propria vita. In Albania fronteggiano la stessa situazione di quella in Germania. Si sentono costretti a intraprendere un viaggio per salvarsi, a cercare un rifugio. Di nuovo il loro viaggio è motivato dalla paura e da una luce di speranza accesa nell'animo. Stavolta la paura domina. E naturale nella loro situazione e deriva dalla stessa specificità 
dell'emozione in questione. Come afferma Ivan Dimitrijević (2016: 37), "la paura configura una passione «intelligente», collegata alla capacità di immaginare il futuro". Occorre aggiungere che i Rosen sopravvivono alla guerra. Tutti i membri della famiglia, oltre ad Abigail, riescono a evitare il contatto diretto con l'oppressore.

\section{DA DACHAU A VALONA}

Ibrahimi si riferisce apertamente alla ricca tradizione della letteratura concentrazionaria, ma ne inserisce nel testo soltanto alcuni elementi. Diversamente ad esempio dall'approccio di Primo Levi, uno dei massimi esponenti di questo genere, dimostrato nell'opera Se questo è un uomo (1946), la scrittrice albanese non descrive in modo minuzioso le condizioni di vita dei prigionieri, neppure le atrocità che hanno sperimentato. Ciò che accomuna la sua tecnica a quella di Levi è il viaggio verso il lager. Ibrahimi ne fornisce un'immagine scioccante, sottolineando la crudeltà dei soldati tedeschi, le sofferenze delle vittime, i loro dubbi sulla meta che raggiungeranno, l'onnipresenza della morte, dei cadaveri massacrati trattati da oggetti inutili. Nel suo racconto spicca il dolore di Abigail, separata forzatamente dalla famiglia, caricata su camion e deportata a Dachau. Visto lo scopo del presente articolo, ci interessa in modo particolare il viaggio che la ragazza compie qualche tempo dopo, ossia quello di ritorno in Albania. Il tema del ritorno è rintracciabile anche in altre opere di autori migranti italofoni, ad esempio nel romanzo Eduart (2005) di Spanjolli. Il suo carattere vi è però completamente diverso. Il protagonista di Spanjolli decide di confrontarsi con la terra natale che ha abbandonato volutamente, ha intenzione di resuscitare il passato per essere sicuro della propria identità, sempre problematica nel paese d'adozione. In Ibrahimi il ritorno in Albania marca la vittoria del bene sul male, la salvezza dalla morte, nonché la possibilità di costruirsi una nuova dimensione vitale. Ciò non significa però che sia privo di crucci. Inizialmente la ragazza stenta a parlare, non può comunicare con il mondo esteriore. Tale sua condizione risulta probabilmente dall'impossibilità della testimonianza (Felman \& Laub 1992: 224). L'orrore che ha vissuto la rende muta, non può essere palesato in quanto inesprimibile, indicibile.

Lo stato di Abigail fa pensare anche al fenomeno dell'afasia. Maurice Halbwachs (1969: 108-109) non considera l'afasia come un difetto che risulta da danni neurologici, ma come un disturbo intellettuale serio che impedisce le relazioni tra individuo e gruppo. Chi ne soffre, non riesce a fare uso di parole, perde la naturale capacità mentale di confrontare le proprie esperienze con le esperienze altrui. La protagonista di Ibrahimi ha subito enormi atrocità, mai sperimentate dai personaggi che la circondano. Si potrebbe dunque azzardare l'ipotesi che non ne parli in quanto sia un 
messaggio incomprensibile. Nessuno sarebbe in grado di provare il suo dolore.

Va detto che generalmente Ibrahimi dedica poco spazio alle conseguenze nefaste della deportazione nel campo di concentramento sulla psiche di Abigail. Tuttavia il lettore è ben cosciente che sono considerevoli. Oltre all'esempio succitato, ne costituisce una buona prova il suo funzionamento nelle fasi successive della vita. La parola "camion" sentita accidentalmente e la stessa azione del viaggiare le danno i brividi, generano sfiducia e inquietudine.

Alla stazione trovano un movimento insolito. [...] Le mani di Abigail tremano. Non capisce cosa sta accadendo. Vede che sono tanti quelli che non salgono sul treno perché non passano i controlli. Ricordi spezzati prendono vita nella sua mente. Stringe la mano del figlio e corre verso la carrozza. Viaggiano sino a Fier in uno scompartimento quasi vuoto. (Ibrahimi 2017: 217)

La studiosa americana Cathy Caruth (2016: 11-12) afferma che il trauma è un' esperienza straziante e incontrollata, accompagnata da vari elementi intrusivi, e che appare come risposta a un evento preciso, sottolinea anche la sua dipendenza dai cambiamenti che avvengono nella vita, dallo spostamento in senso spaziale e temporale. Il trauma avviene in un altro luogo e in un altro tempo (Caruth 2016: 18-19). L'atmosfera commossa del viaggio che Abigail ha deciso di compiere diventa un avvenimento catastrofico che suscita ricordi dolorosi, la paralizza in qualche modo. Emmanuel Lévinas (2006: 148-149), analizzando il rapporto tra sofferenza e tempo, arriva alla conclusione che non è possibile rimediare alla sofferenza vissuta in passato. Per risarcire i danni psichici subiti, si dovrebbe rivivere la situazione portatrice di dolore e il presente non offre tale opportunità. L'individuo non potrà approfittarne che nell'eternità, dunque dopo il trapasso. Dall'altro lato il presente non può redimersi dai ricordi. La protagonista risulta una figura infelice, condannata a vivere all'insegna dello spettro del trauma e della sofferenza, che nel periodo subito dopo il ritorno in Albania, la mettono in uno stato di incomunicabilità, negli anni successivi si manifestano attraverso le reazioni a situazioni concrete. I suoi ricordi sono popolati da più avvenimenti dove spiccano le figure dei membri della famiglia. Abigail ricorda in modo spontaneo, a contatto con vari elementi. Una volta si tratta del tema che viene toccato, un'altra volta è un oggetto particolare che le si trova vicino. Il concetto proustiano di memoria involontaria funge a Ibrahimi da mezzo per tradurre la nostalgia dei genitori e della sorella che la protagonista prova nell'animo. Dalla prospettiva del lettore, a un certo punto, l'assenza di questi personaggi nel suo spazio vitale si rivela più dolorosa delle esperienze vissute nel lager. Abigail è capace di riflettere sulle loro figure, di resuscitare i momenti trascorsi insieme, di esprimere quanto le manchino, 
non risulta invece in grado, visto l'effetto che le hanno provocato il trauma e la sofferenza, di parlare della deportazione in Germania.

L'attenzione di Ibrahimi si focalizza di nuovo sui cambiamenti che il viaggio introduce nella vita. In Albania Abigail, sebbene inizialmente abbia difficoltà nel fidarsi di altri e nel comunicare, riesce a stringere rapporti durevoli e felici con le persone in cui si imbatte. Il microcosmo di cui diventa parte integrante, col tempo si dimostra amichevole e le consente di gustare la normalità. Il romanzo fornisce informazioni sulle sue vicende esistenziali nel periodo che va dall'infanzia fino all'età adulta. Il suo ritorno e le riflessioni sulla sua condizione servono a presentare alcuni elementi della realtà sociopolitica del paese nel secondo dopoguerra. Si tratta di un quadro coerente, tratteggiato per sottolineare come funzioni il comunismo. La protagonista conosce molto bene le regole imposte dal sistema, che penetrano nella vita dell'uomo, dandogli l'impressione di essere continuamente controllato. Le aspirazioni individuali vanno soffocate e conta soltanto il bene collettivo. Ibrahimi, come Dones (1997), Guaci (2005) oppure Kubati (2000), denuncia l'ingiustizia diffusa ai tempi del regime di Hoxha. La sua protagonista lo rifiuta in modo aperto. Prende la decisione molto spontanea di lasciare il paese, di fuggire. Non riflette sulle conseguenze, sulla reazione del marito, si presenta come una madre preoccupata per il futuro dei figli che porta con sé. In Albania non sarà possibile il loro sviluppo. Si potrebbe azzardare l'ipotesi che la sua decisione sia motivata anche dal desiderio di ritrovare la sorella, oppure che senta il bisogno di recarsi altrove per guarire dal trauma. Di sicuro va in Occidente, non si sa però dove esattamente.

La vita di Abigail diventa un viaggio continuo, uno spostamento intrigante alla ricerca della normalità, di prospettive migliori. Il suo ritorno in Albania si trasforma in una partenza imprevista, in un nuovo viaggio, che di sicuro avrà un impatto enorme sul suo futuro.

\section{CONCLUSIONI}

Ibrahimi delinea un'immagine poliprospettica e molto originale del fenomeno del viaggio nel panorama della letteratura della migrazione. In primo luogo, lo associa all'esperienza dell'Olocausto. In secondo luogo, presenta lo spostamento fisico, la cui meta costituisce la terra albanese, mostrata in un'ottica positiva, e rovescia lo schema che domina nelle opere dei suoi connazionali italofoni. I protagonisti si recano in Albania per salvarsi dalla morte, trovare ospitalità, cordialità, nonché il tempo necessario per distanziarsi dalla quotidianità e fare i conti con il passato. Si immergono anche nella storia e nella cultura del paese che sembra loro esotico e a volte suscita ribrezzo. Vi si costruiscono il nuovo spazio vitale, si integrano nella società locale, in un certo senso, si sottopongono volutamente al processo 
di albanizzazione, esteso addirittura alla lingua, che cercano di saper capire e usare in qualche maniera.

In Ibrahimi l'Albania diventa la terra promessa, dove l'individuo ottiene la possibilità di realizzare gli scopi essenziali nella sua esistenza. Non si può però non menzionare il fatto che ogni tanto il suo cammino è segnato da emozioni tumultuose, come il senso di mancanza, la nostalgia, il trauma e la paura, le quali risultano da condizionamenti personali specifici.

\section{BIBLIOGRAFIA}

Ali Farah, C. (2007). Madre piccola. Segarate: Frassinelli.

Augé, M. (2009). Formy zapomnienia. Kraków: Universitas.

Caruth, C. (2016). Unclaimed experience, Trauma, Narrative, and History. Baltimore: Johns Hopkins University Press.

Coman, I. B. (2012). Dodici più un angelo. Murazzano: Ellin Selae.

Comberiati, D. \& Bond, E. (a cura di) (2013). Il confine liquido. I rapporti letterari e interculturali fra Italia e Albania. Nardò: Besa.

Crainz, G. (2007). L'ombra della guerra. Il 1945 l'Italia. Roma: Donzelli.

Dimitrijević, I. (2016). La contraffazione della politica. La paura della fine, la tutela del bios e il potere della socializzazione. Saonara: Il prato.

Dones, E. (1997). Senza bagagli. Nardò: Besa.

Dones, E. (2007). Vergine giurata. Milano: Feltrinelli.

Felman, S. \& Laub, D. (1992). Testimony: Crises of Witnessing in Literature, Psychoanalysis and History. New York: Routledge.

Guaci, L. (1999). La panciera rossa. Roma: Stagno Editore.

Guaci, L. (2005). I grandi occhi del mare. Nardò: Besa.

Halbwachs, M. (1969). Społeczne ramy pamięci. Warszawa: Państwowe Wydawnictwo Naukowe.

Henderson, J. (2005). Pamięć i zapominanie. Gdańsk: Gdańskie Wydawnictwo Psychologiczne.

Ibrahimi, A. (2008). Rosso come una sposa. Torino: Einaudi.

Ibrahimi, A. (2017). Il tuo nome è una promessa. Torino: Einaudi.

Kubati, R. (2000). Va e non torna. Nardò: Besa.

Kubati, R. (2002). M. Nardò: Besa.

Lakhous, A. (2006). Lo scontro di civiltà per un ascensore a piazza Vittorio. Roma: Edizioni e/o.

Lakhous, A. (2010). Divorzio all 'islamica a viale Marconi. Roma: Edizioni e/o.

Levi, P. (1946). Se questo è un uomo. Torino-Firenze: Edizioni De Silva.

Lévinas, E. (2006). Istniejacy i istnienie. Kraków: Homini.

Lovecraft, H. P. (1969). Epouvante et surnaturel en littérature. Paris: Christian Bourgois Éditeur. 
Serkowska, H. (2012). Dopo il romanzo storico. La storia nella letteratura italiana del '900. Pesaro: Metauro.

Spanjolli, A. (2005). Eduart. Nardò: Besa.

Spanjolli, A. (2007). L'accusa silenziosa. Nardò: Besa.

Spanjolli, A. (2012). I nipoti di Scanderbeg. Nardò: Besa.

Vorpsi, O. (2005). Il paese dove non si muore mai. Torino: Einaudi.

Vorpsi, O. (2007). La mano che non mordi. Torino: Einaudi.

White, H. (2010). Poetyka pisarstwa historycznego. Kraków: Universitas.

\section{A JOURNEY TO ALBANIA: ANILDA IBRAHIMI'S \\ IL TUO NOME È UNA PROMESSA}

\section{Summary}

The article analyzes the travel motif in the latest novel by Anilda Ibrahimi, an Albanian author writing in Italian. The essay is divided into three sections that reflect the destinations of the novel's characters: Tirana, Kavajë and Valona, all located in Albania. The article discusses various aspects of travel and travel writing: destinations, finding oneself in a new reality and the way it influences their actions. Travel proves to have great significance; it helps to assemble information concerning history and culture, reflect on the past, and even avoid death. Albania is depicted in a positive way, as a country with hope for a better future, in spite of the occasional pain.

Keywords: Italy, Albania, journey, hospitality, culture, self-consciousness. 